\title{
Computed tomography Hounsfield unit-based prediction of pedicle screw loosening after surgery for degenerative lumbar spine disease
}

\author{
*Da Zou, MD, Aikeremujiang Muheremu, MD, PhD, Zhuoran Sun, MD, Woquan Zhong, MD, \\ Shuai Jiang, MD, and Weishi Li, MD
}

Department of Orthopedics, Peking University Third Hospital, Beijing, China

\begin{abstract}
OBJECTIVE The authors investigated the relation between Hounsfield unit $(\mathrm{HU})$ values measured on $\mathrm{CT}$ and the risk of pedicle screw loosening in patients who underwent lumbar pedicle screw fixation for degenerative lumbar spine disease. METHODS Patients who were treated with lumbar pedicle screw fixation between July 2011 and December 2015 at the authors' department were reviewed. Age, sex, BMI, smoking and diabetes histories, range of fixation, and fusion method were recorded as the basic patient information. The HU values for lumbar bone mineral density (BMD) for the L1, L2, L3, and L4 vertebra were measured on CT scans. Logistic regression analysis was used to identify the independent influencing factors of pedicle screw loosening.
\end{abstract}

RESULTS A total of 503 patients were included in the final analysis. The pedicle screw loosening rate at the 12-month follow-up was $30.0 \%$ (151 of 503 patients). There were no significant differences in sex, BMI, or histories of smoking and diabetes between the patients with (loosening group) and those without (nonloosening group) screw loosening $(p>0.05)$. The mean $\mathrm{HU}$ value of $\mathrm{L} 1-4$ was lower in the loosening group than the nonloosening group (106.3 $\pm 33.9 \mathrm{vs} 132.6 \pm$ $42.9, \mathrm{p}<0.001)$. In logistic regression analysis, being male (OR $2.065 ; 95 \% \mathrm{Cl} 1.242-3.433)$, $\mathrm{HU}$ value (OR $0.977 ; 95 \%$ $\mathrm{Cl} 0.970-0.985$ ), length of fixation (OR 3.616; $95 \% \mathrm{Cl} 2.617-4.996)$, and fixation to S1 (OR 1.699; 95\% Cl 1.039-2.777) were the independent influencing factors for screw loosening.

CONCLUSIONS HU value measured on CT was an independent predictor for pedicle screw loosening, and lower HU value was significantly correlated with higher risk of screw loosening.

https://thejns.org/doi/abs/10.3171/2019.11.SPINE19868

KEYWORDS pedicle screw loosening; risk factors; computed tomography; Hounsfield units; osteoporosis; lumbar; degenerative

$\mathrm{W}$ ITH aging of the population, osteoporosis has gradually become a global health problem that affects 200 million people worldwide. ${ }^{6}$ It has been estimated that there will be more than 400 million elderly people and 200 million osteoporotic patients in China by the year $2050.4,13$ An increasing number of elderly patients now need surgical treatment due to degenerative lumbar diseases such as degenerative lumbar spinal stenosis, degenerative lumbar spondylolisthesis, and degenerative scoliosis. ${ }^{5}$ Lumbar surgeries are performed 3 times more frequently in patients $\geq 60$ years old than in younger patients. ${ }^{24}$ The rate of osteoporosis in older patients is re- ported to be higher than that of the general population, with a rate of $50 \%$ in older female patients. ${ }^{5}$

Pedicle screw fixation is a common procedure used in the surgical treatment of lumbar degenerative diseases, while the osteoporotic lumbar spine has created great challenges in maintaining the stability of lumbar fixation. Pedicle screw loosening is one of the main reasons for secondary surgical interventions after lumbar surgery, and osteoporosis, or low bone mineral density (BMD), is the most frequently reported risk factor for screw loosening. ${ }^{10}$ Although the reported incidence of screw loosening varies among different published studies, in patients with

ABBREVIATIONS BMD = bone mineral density; DXA = dual-energy $\mathrm{x}$-ray absorptiometry; HU = Hounsfield units; LIV = lowest instrumented vertebra; ODI = Oswestry Disability Index; PLF = posterolateral lumbar fusion; PLIF = posterior lumbar interbody fusion; ROI = region of interest; VAS = visual analog scale.

SUBMITTED July 28, 2019. ACCEPTED November 6, 2019.

INCLUDE WHEN CITING Published online January 3, 2020; DOI: 10.3171/2019.11.SPINE19868.

* D.Z. and A.M. contributed equally to this work. 
osteoporosis the risk of screw loosening is twice that of patients without osteoporosis, and $6.3 \%-15.6 \%$ of patients with screw loosening require revision surgery. ${ }^{3,8,20} \mathrm{Howev-}$ er, only a handful of clinical studies have reported on the relation between low BMD and pedicle screw loosening. ${ }^{8}$

BMD measured in Hounsfield units (HU) on CT is used in the evaluation of BMD and can serve as a diagnostic tool for osteoporosis. Although there are reports of significant correlations between $\mathrm{HU}$ values and measurements obtained with dual-energy x-ray absorptiometry (DXA), few studies have been carried out on the application of HU values to prediction of pedicle screw loosening. ${ }^{2,27}$ In the current study, to provide reference value information for future clinical practice, we retrospectively reviewed patients who received surgical intervention with pedicle screw fixation for degenerative lumbar disease, and we investigated possible correlations between $\mathrm{HU}$ values and the incidence of pedicle screw loosening in these patients.

\section{Methods}

\section{Patients}

This study was approved by the Ethical Committee of our hospital (IRB00006761-M2018012); the requirement for informed consent was waived because this was a retrospective study. Patients who were surgically treated by the same spine surgeon group in our department for lumbar degenerative diseases between July 2011 and December 2015 were retrospectively included in the current study. Included patients met the following criteria: 1) age 50 years or older; 2) underwent surgical treatment with lumbar pedicle screw fixation; 3) underwent CT scans of the lumbar spine within 3 months before surgery; and 4) underwent follow-up for at least 12 months. Exclusion criteria were the following: 1) previous history of spinal surgery; 2) presence of congenital scoliosis, spinal fracture, spine tumor, or ankylosing spondylitis; 3) $>4$ levels of fixation; 4) level of the lowest instrumented vertebra (LIV) higher than L5; 5) presence of other circumstances that affect bone metabolism, such as long-term steroid use; 6) occurrence of an unsuccessful attempt to place a screw at an appropriate position in a single try, such as the placement of a screw that causes injury to the pedicle wall; and 7) history of reoperation within a year after initial surgery for reasons other than screw loosening, such as infection, hematoma, neural injury, or adjacent level disk degeneration. General patient information was recorded, including age, sex, BMI, and histories of smoking and diabetes. At the 12-month follow-up, patients with and those without screw loosening were divided into 2 groups, the loosening group and the nonloosening group.

\section{Surgical Technique}

All surgery was performed with an open, posterior midline approach by the same surgical team specializing in spinal disorders. Patients underwent either posterolateral lumbar fusion (PLF) or posterior lumbar interbody fusion (PLIF), both instrumented with conventional pedicle screw fixation (screw diameter $6.0-6.5 \mathrm{~mm}$ for lumbar vertebrae, $7.0 \mathrm{~mm}$ for $\mathrm{S} 1$; length $40-45 \mathrm{~mm}$ for lumbar vertebrae, $35-40 \mathrm{~mm}$ for $\mathrm{S} 1$ ). The starting point for ped- icle screw insertion was at the intersection of the midline of the transverse process and the sagittal plane through the lateral margin of the superior facet. The orientation of screw insertion was determined by reference to preoperative and intraoperative radiographs. For PLIF procedures, a PEEK cage packed with autogenous bone was placed into the interbody space following adequate decompression, discectomy, and endplate decortication. The autograft was harvested from bone removed for decompression. To simplify the data analysis, we divided the patients into 2 groups: patients who underwent PLIF at the caudal segment (the PLIF group) and patients who underwent PLF alone at the caudal segment (the non-PLIF group).

After surgery, the patients were instructed to walk by themselves with a lumbar support brace after 1-3 days of bed rest and to wear the brace for 2-3 months. Only essential daily activities were allowed for the first month after surgery. Physical therapy such as ankle pump exercise was started the first day after the surgery, and the types and frequency of exercise depended on the degree of recovery.

\section{Measurements of HU Value}

All of the patients underwent a preoperative lumbar CT scan (Definition, Siemens). The tube voltage of all of the CT scans was set at $120 \mathrm{kV}$. An experienced spine surgeon (D.Z.) independently used a PACS system (GE Electrics) to measure the $\mathrm{HU}$ value. At a horizontal plane at the middle level of the vertebral body, a region of interest (ROI) was chosen, avoiding cortical bone and the area of posterior venous plexus. The mean HU value in the ROI was measured automatically by PACS (Fig. 1). In line with the DXA method, we used the mean BMD of L1-4 to represent the overall BMD of the lumbar spine when we calculated the mean $\mathrm{HU}$ value of L1-4.

\section{Outcome Assessment}

At the 12-month follow-up, lumbar radiographs were obtained for all patients. Another senior spine surgeon (W.Z.) independently evaluated screw loosening and bone fusion. Screw loosening was diagnosed when the clear zone around the pedicle screw exceeded $1 \mathrm{~mm}$ (Fig. 2). Pseudarthrosis was diagnosed when segmental translation of $\geq 3 \mathrm{~mm}$ or rotation of $\geq 3^{\circ}$ was observed in lateral flexion-extension radiographs or there was no continued bone fusion mass at any fusion segment at the 12-month followup. Clinical outcomes were assessed during follow-up using a visual analog scale (VAS; range $0-10$ ) and Oswestry Disability Index (ODI) scores (range 0\%-100\%).

\section{Statistical Analysis}

All data were analyzed using SPSS 23.0 (IBM Corporation). Continuous variables were recorded as means \pm standard deviations. Quantitative measures were recorded as percentages. Independent sample t-tests were applied to compare the variables between groups. Quantitative data were analyzed with the chi-square or Fisher's exact test. Logistic regression analysis was fitted for the outcomes, and the results were presented as odds ratios (ORs) with $95 \%$ confidence intervals (CIs); $\mathrm{p}<0.05$ was considered to indicate a statistically significant difference. 

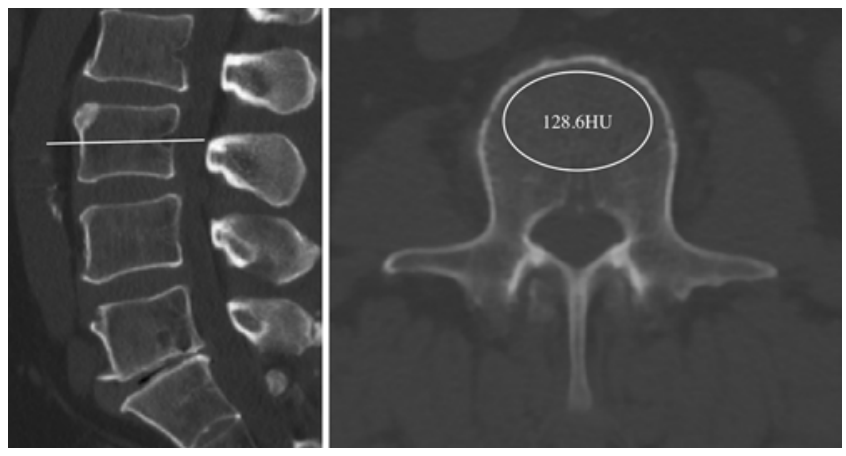

FIG. 1. Application of the CT plus PACS system to measure the HU values of the lumbar spine. The HU value of 128.6 on the horizontal plane of the L 3 vertebra is shown.

\section{Results}

A total of 503 patients were included in the final analysis (188 male and 315 female patients; mean age $61.2 \pm$ 6.7 years, range 50-83 years) with a mean BMI of 25.9 $\pm 3.3 \mathrm{~kg} / \mathrm{m}^{2}$. At the 12-month follow-up, the overall rate of screw loosening was $30.0 \%$ (151/503). The screw loosening rate increased with the number of fixation levels and was $4.1 \%$ (7/170), 33.3\% (70/210), 53.3\% (48/90), and $78.8 \%(26 / 33)$ for 1 level, 2 levels, 3 levels, and 4 levels of fixation, respectively $(\mathrm{p}<0.001)$. The loosening rate was higher in the patients with LIV at S1 than in the patients with LIV at L5 (39.9\% vs $23.3 \%$, p < 0.001). There were 323 patients who underwent PLIF at the caudal segment (PLIF group); the remaining 180 patients underwent PLF alone at the caudal segment (non-PLIF group). The PLIF patients showed lower pedicle screw loosening rates than the non-PLIF patients $(22.9 \%$ vs $42.8 \%, \mathrm{p}<0.001)$.

The rate of pseudarthrosis was higher in the patients with screw loosening (loosening group) than in those without (nonloosening group) $(43.0 \%$ vs $2.6 \%, \mathrm{p}<0.001)$. The preoperative leg pain VAS and the ODI scores were higher in the loosening group (leg pain VAS scores $6.0 \pm$ 2.0 vs $5.5 \pm 2.3$, ODI scores $37.2 \pm 18.9$ vs $33.0 \pm 17.8, p$ $<0.05$ ), while the preoperative low-back pain VAS scores showed no significant difference between the 2 groups (low-back pain VAS score $5.1 \pm 2.2$ vs $5.0 \pm 2.1$, p >0.05). The postoperative low-back pain VAS scores were higher in the loosening group (low-back pain VAS scores $2.9 \pm$ 2.1 vs $2.5 \pm 2.1, \mathrm{p}<0.05$ ), while the postoperative leg pain VAS and ODI scores showed no significant differences between the 2 groups (leg pain VAS scores $2.3 \pm 2.3$ vs $2.1 \pm 2.2$, ODI scores $22.9 \pm 17.3$ vs $19.9 \pm 15.3, \mathrm{p}>0.05$ ).

The comparison of general characteristics between the loosening and nonloosening groups is shown in Table 1. The sex, BMI, and histories of smoking and diabetes showed no significant differences between the 2 groups $(\mathrm{p}>0.05)$. However, there were significant differences between the 2 groups in age, vertebral HU value of L1-4, length of fixation, level of LIV (S1 vs L5), and the fusion method of the caudal segment (PLIF vs non-PLIF). Specifically, the mean HU value of L1-4 was much lower in the loosening group than the nonloosening group $(106.3 \pm$ 33.9 vs $132.6 \pm 42.9, \mathrm{p}<0.001)$. The factors with a $\mathrm{p}$ value

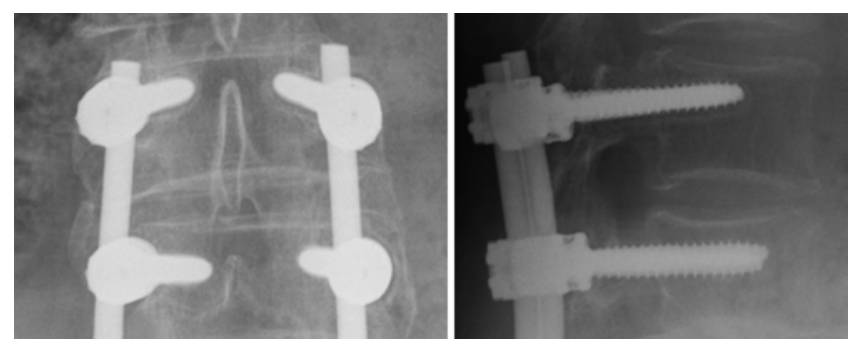

FIG. 2. Anteroposterior (left) and lateral (right) radiographs of a patient with postoperative screw loosening.

of $<0.1$ in Table 1 were chosen as potential contributing factors for screw loosening and included in the logistic regression analysis. The further logistic regression analysis showed that male sex (OR 2.065; 95\% CI 1.242-3.433), mean HU value of L1-4 (OR 0.977; 95\% CI 0.970-0.985), length of fixation (OR 3.616; 95\% CI 2.617-4.996), and fixation to S1 (OR 1.699; 95\% CI 1.039-2.777) were the independent influencing factors for screw loosening (Table 2).

\section{Discussion}

The most commonly used parameter for the evaluation of BMD is DXA. The lumbar spine (L1-4) and proximal femoral bone are the main sites for measurement, and the diagnosis of osteoporosis is normally made when the lowest T-score is $<-2.5 .{ }^{23}$ However, the accuracy of DXAbased assessment of BMD is affected by variables such as lumbar degenerative changes, spinal deformity, previous spinal surgery, vertebral compressive fractures, etc. ${ }^{28}$

TABLE 1. Comparison of patient characteristics between the pedicle screw loosening and nonloosening groups

\begin{tabular}{|c|c|c|c|}
\hline & Loosening & Nonloosening & $p$ Value \\
\hline Patients & 151 & 352 & \\
\hline Age, yrs & $63.5 \pm 6.4$ & $60.2 \pm 6.6$ & $<0.001$ \\
\hline Sex & & & 0.085 \\
\hline Male & 65 & 123 & \\
\hline Female & 86 & 229 & \\
\hline BMI, $\mathrm{kg} / \mathrm{m}^{2}$ & $25.8 \pm 3.2$ & $25.9 \pm 3.4$ & 0.937 \\
\hline Smoker & 24 & 48 & 0.508 \\
\hline Diabetes & 24 & 57 & 0.933 \\
\hline LIV at S1 & 81 & 122 & $<0.001$ \\
\hline PLIF at caudal segment & 74 & 249 & $<0.001$ \\
\hline \multicolumn{4}{|l|}{ HU value } \\
\hline L1 & $114.8 \pm 34.9$ & $143.7 \pm 46.3$ & $<0.001$ \\
\hline L2 & $108.1 \pm 36.4$ & $134.9 \pm 45.4$ & $<0.001$ \\
\hline L3 & $100.1 \pm 35.5$ & $125.6 \pm 43.1$ & $<0.001$ \\
\hline L4 & $102.2 \pm 36.0$ & $125.9 \pm 42.7$ & $<0.001$ \\
\hline Mean L1-4 & $106.3 \pm 33.9$ & $132.6 \pm 42.9$ & $<0.001$ \\
\hline
\end{tabular}

Values are presented as number of patients unless otherwise indicated. Mean values are presented \pm SD. 
TABLE 2. Results of logistic regression analysis of factors influencing screw loosening after lumbar surgery

\begin{tabular}{lcr}
\hline & OR $(95 \% \mathrm{Cl})$ & p Value \\
\hline Age in yrs & $1.034(0.994-1.076)$ & 0.098 \\
\hline Male sex & $2.065(1.242-3.433)$ & 0.005 \\
\hline Length of fixation & $3.616(2.617-4.996)$ & $<0.001$ \\
\hline Mean HU value of L1-4 & $0.977(0.970-0.985)$ & $<0.001$ \\
\hline Fixation to S1 & $1.699(1.039-2.777)$ & 0.035 \\
\hline PLIF of the caudal segment & $0.773(0.471-1.267)$ & 0.307 \\
\hline
\end{tabular}

Pickhardt et al. reported that $52.1 \%$ of patients with vertebral compressive fracture had false-negative results when DXA was used to detect osteoporosis. ${ }^{18}$ The most accurate site to assess BMD is the cancellous bone. ${ }^{11,14}$ Quantitative CT (QCT)-based evaluation of BMD has been gaining popularity over the last few years. Although QCT can accurately focus on the cancellous bone and be used to make early and accurate diagnoses of osteoporosis, the high cost of the equipment and need for rigorous training to use the QCT software currently make it difficult to apply this technique in many clinical settings. ${ }^{2}$

Unlike DXA, the ROI of the HU is placed in the cancellous area of the vertebral body, which makes the HU value less affected by lumbar degenerative changes. ${ }^{28}$ It has been widely reported that $\mathrm{HU}$ value-based evaluation of BMD is correlated with the standard BMD value and physical strength of the bone. ${ }^{18,22}$ The vertebral HU value also has excellent validity in detecting osteoporosis and good interexamination and interobserver reliability. ${ }^{19}$ In addition, considering that most of the patients requiring lumbar surgery undergo preoperative lumbar CT scans, the measurement of CT HU values can offer more BMD information with no extra cost or additional radiation exposure. Thus the CT HU value is theoretically a useful predictor for BMD-related complications after lumbar surgery. However, few studies have investigated its use in this setting.

Pedicle screw loosening is one of the most common complications of lumbar spine surgery. Considering the fact that there is no standard method to diagnose screw loosening, in this study we used the clear zone around screws as the diagnostic criterion. It has been reported that when the clear zone around a screw is $\geq 1 \mathrm{~mm}$ there is $64 \%-89 \%$ sensitivity and $84 \%-100 \%$ specificity for the diagnosis of screw loosening. ${ }^{21,25} \mathrm{We}$ found that the overall screw loosening rate was $30.0 \%$ at the 12 -month follow-up. The incidence of screw loosening varies widely among previous studies. ${ }^{8}$ This could be due to differences in the primary diseases, methods of surgery, length of fixation, lumbar BMD, and/or time of follow-up, as well as differences in statistical approaches. For example, the incidence of screw loosening can be as high as $60 \%$ after multilevel fixation or in patients with osteoporotic vertebral fractures.? Tokuhashi et al. reported that the screw loosening rate was only $7.4 \%$ for 1-level fixation, while it was over $40 \%$ in patients with 4 levels of fixation at $\geq 3$ years follow-up. ${ }^{26}$
In the current literature, the most frequently reported risk factors for screw loosening include older age, low BMD, and multilevel fixation, as well as fixation of S1, and the latter 3 of these factors were also identified as independent risk factors in our study. ${ }^{3,12,16,26}$ Although the loosening and nonloosening groups had significant differences in patient age, patient age was not an independent risk factor in our study according to the logistic regression analysis. A possible reason for this is that BMD decreases with age, and the loosening rate was higher in the older patients mainly because of their lower BMD rather than their age itself. ${ }^{4}$ The effect of sex on screw loosening is still controversial. ${ }^{1,17}$ Although female patients usually have lower BMDs than male patients, we found that being male was an independent risk factor. ${ }^{5}$ This result could be attributable to higher amounts of manual labor and other physical activities performed by men than women, which increases the load on the fixation construct. The length of fixation is a frequently mentioned predictor of screw loosening. In the literature, the reported loosening rate was usually lower than $10 \%$ for 1-level lumbar fusion with pedicle screw fixation, but the rate increased significantly when the length of fixation was $\geq 2$ levels. ${ }^{12,17,26}$ In another investigation of single-level fixation, Mo et al. found no differences in the screw loosening and fusion rates between the cement-augmented pedicle screw group and the conventional screw group..$^{15}$ Fixation to $\mathrm{S} 1$ is also a significant contributor to screw loosening, especially for the S1 screws. ${ }^{12}$ In comparison with the lumbar vertebrae, the $\mathrm{S} 1$ pedicle is shorter and wider and the S1 vertebral body is smaller. These anatomical factors may account for the high rate of screw loosening in patients with fixation to S1. In addition, the risk of screw loosening is associated with the fusion method. ${ }^{8}$ Lumbar interbody fusion with a cage offers anterior support and reduced load on the pedicle screws. However, the fusion method used in a single patient can be very complex, such as the combination of PLF and PLIF at different lumbar segments. Given that the caudal segment is the most common site for performing PLIF and that screw loosening occurs most often at the LIV, we focused only on the fusion method at the caudal segment to simplify the analysis. ${ }^{9}$ The results showed that the PLIF group had a lower loosening rate than the non-PLIF group (22.9\% vs 42.8\%), but the use of PLIF was not an independent factor influencing screw loosening. This result could indicate that the fusion method is less important than other independent factors in the development of pedicle screw loosening.

Although previous studies have suggested that lower BMD is a risk factor for screw loosening, these studies lacked logistic regression analyses that included other main contributing factors. ${ }^{16,26}$ Our results demonstrated that the patients with screw loosening had lower HU values of only about $110 \mathrm{HU}$ on average, which was very close to the mean $\mathrm{HU}$ value in osteoporotic patients..$^{18,28}$ Moreover, in the logistic regression analysis in our study the $\mathrm{HU}$ value was proven to be an independent predictor for screw loosening. Considering that DXA T-scores can be falsely elevated by lumbar degeneration, we recommend using CT HU values as a complement to DXA T-scores for BMD evaluation during surgical planning. Lumbar spine $\mathrm{CT}$ is a common and simple procedure for patients before 
surgical treatment, making it easier to apply the CT HU method in most medical centers.

The main objective for pedicle screw fixation is to facilitate fusion. However, clinical data are scarce regarding the correlation between screw loosening and bone fusion. Tokuhashi et al. found that one-half of the patients with sustained screw loosening observed on radiographs had pseudarthrosis. ${ }^{26}$ In our study, the rate of pseudarthrosis was up to $43.0 \%$ in the screw loosening group and was only $2.6 \%$ in the nonloosening group. These findings further demonstrated that pedicle screw loosening was highly correlated with fusion failure.

\section{Study Limitations}

There are several limitations of this study. First, the ROIs for HU measurements were chosen manually, which could raise concerns over the repeatability of those results. However, it has been proven that HU measurements have excellent interobserver and intraobserver reliability. ${ }^{19,29}$ Second, this was a retrospective analysis, and additional prospective studies are needed to examine the correlation between HU values and screw loosening risk. Third, we analyzed only the most commonly reported factors. Some other potential influencing factors, such as pedicle screw size, postoperative sagittal alignment, and spinopelvic parameters, were not included in the statistical analysis. ${ }^{12}$

\section{Conclusions}

The HU value was significantly correlated with the incidence of screw loosening and can be used as an independent factor to predict screw loosening in patients requiring pedicle screw fixation for the treatment of degenerative lumbar spine disease.

\section{Acknowledgments}

This work was supported by the Clinical Cohort Construction Program of Peking University Third Hospital, China National Key Research and Development Program (2018YFB1307700), and the Natural Science Foundation of China (8187180 and 81860235).

\section{References}

1. Abul-Kasim K, Ohlin A: Evaluation of implant loosening following segmental pedicle screw fixation in adolescent idiopathic scoliosis: a 2 year follow-up with low-dose CT. Scoliosis 9:13, 2014

2. Anderson PA, Polly DW, Binkley NC, Pickhardt PJ: Clinical use of opportunistic computed tomography screening for osteoporosis. J Bone Joint Surg Am 100:2073-2081, 2018

3. Bredow J, Boese CK, Werner CML, Siewe J, Löhrer L, Zarghooni K, et al: Predictive validity of preoperative CT scans and the risk of pedicle screw loosening in spinal surgery. Arch Orthop Trauma Surg 136:1063-1067, 2016

4. Chen P, Li Z, Hu Y: Prevalence of osteoporosis in China: a meta-analysis and systematic review. BMC Public Health 16:1039, 2016

5. Chin DK, Park JY, Yoon YS, Kuh SU, Jin BH, Kim KS, et al: Prevalence of osteoporosis in patients requiring spine surgery: incidence and significance of osteoporosis in spine disease. Osteoporos Int 18:1219-1224, 2007

6. Cotts KG, Cifu AS: Treatment of osteoporosis. JAMA 319:1040-1041, 2018
7. El Saman A, Meier S, Sander A, Kelm A, Marzi I, Laurer $\mathrm{H}$ : Reduced loosening rate and loss of correction following posterior stabilization with or without PMMA augmentation of pedicle screws in vertebral fractures in the elderly. Eur J Trauma Emerg Surg 39:455-460, 2013

8. Galbusera F, Volkheimer D, Reitmaier S, Berger-Roscher N, Kienle A, Wilke HJ: Pedicle screw loosening: a clinically relevant complication? Eur Spine J 24:1005-1016, 2015

9. Gazzeri R, Roperto R, Fiore C: Surgical treatment of degenerative and traumatic spinal diseases with expandable screws in patients with osteoporosis: 2-year follow-up clinical study. J Neurosurg Spine 25:610-619, 2016

10. Goldstein CL, Brodke DS, Choma TJ: Surgical management of spinal conditions in the elderly osteoporotic spine. Neurosurgery 77 (Suppl 4):S98-S107, 2015

11. Hamdy RC, Petak SM, Lenchik L: Which central dual X-ray absorptiometry skeletal sites and regions of interest should be used to determine the diagnosis of osteoporosis? J Clin Densitom 5 Suppl:S11-S18, 2002

12. Kim JB, Park SW, Lee YS, Nam TK, Park YS, Kim YB: The effects of spinopelvic parameters and paraspinal muscle degeneration on S1 screw loosening. J Korean Neurosurg Soc 58:357-362, 2015

13. Lin X, Xiong D, Peng YQ, Sheng ZF, Wu XY, Wu XP, et al: Epidemiology and management of osteoporosis in the People's Republic of China: current perspectives. Clin Interv Aging 10:1017-1033, 2015

14. Lorentzon M, Cummings SR: Osteoporosis: the evolution of a diagnosis. J Intern Med 277:650-661, 2015

15. Mo GY, Guo HZ, Guo DQ, Tang YC, Li YX, Yuan K, et al: Augmented pedicle trajectory applied on the osteoporotic spine with lumbar degenerative disease: mid-term outcome. J Orthop Surg Res 14:170, 2019

16. Okuyama K, Abe E, Suzuki T, Tamura Y, Chiba M, Sato K: Influence of bone mineral density on pedicle screw fixation: a study of pedicle screw fixation augmenting posterior lumbar interbody fusion in elderly patients. Spine J 1:402-407, 2001

17. Orita S, Ohtori S, Eguchi Y, Kamoda H, Arai G, Ishikawa T, et al: Radiographic evaluation of monocortical versus tricortical purchase approaches in lumbosacral fixation with sacral pedicle screws: a prospective study of ninety consecutive patients. Spine (Phila Pa 1976) 35:E1230-E1237, 2010

18. Pickhardt PJ, Pooler BD, Lauder T, del Rio AM, Bruce RJ, Binkley N: Opportunistic screening for osteoporosis using abdominal computed tomography scans obtained for other indications. Ann Intern Med 158:588-595, 2013

19. Pompe E, de Jong PA, de Jong WU, Takx RAP, Eikendal ALM, Willemink MJ, et al: Inter-observer and inter-examination variability of manual vertebral bone attenuation measurements on computed tomography. Eur Radiol 26:30463053, 2016

20. Röllinghoff M, Schlüter-Brust K, Groos D, Sobottke R, Michael JWP, Eysel P, et al: Mid-range outcomes in 64 consecutive cases of multilevel fusion for degenerative diseases of the lumbar spine. Orthop Rev (Pavia) 2:e3, 2010

21. Sandén B, Olerud C, Petrén-Mallmin M, Johansson C, Larsson $\mathrm{S}$ : The significance of radiolucent zones surrounding pedicle screws. Definition of screw loosening in spinal instrumentation. J Bone Joint Surg Br 86:457-461, 2004

22. Schreiber JJ, Anderson PA, Rosas HG, Buchholz AL, Au AG: Hounsfield units for assessing bone mineral density and strength: a tool for osteoporosis management. J Bone Joint Surg Am 93:1057-1063, 2011

23. Shepherd JA, Schousboe JT, Broy SB, Engelke K, Leslie WD: Executive Summary of the 2015 ISCD Position Development Conference on advanced measures from DXA and QCT: fracture prediction beyond BMD. J Clin Densitom 18:274-286, 2015 
24. Sivasubramaniam V, Patel HC, Ozdemir BA, Papadopoulos MC: Trends in hospital admissions and surgical procedures for degenerative lumbar spine disease in England: a 15-year time-series study. BMJ Open 5:e009011, 2015

25. Spirig JM, Sutter R, Götschi T, Farshad-Amacker NA, Farshad M: Value of standard radiographs, computed tomography, and magnetic resonance imaging of the lumbar spine in detection of intraoperatively confirmed pedicle screw loosening-a prospective clinical trial. Spine J 19:461-468, 2019

26. Tokuhashi Y, Matsuzaki H, Oda H, Uei H: Clinical course and significance of the clear zone around the pedicle screws in the lumbar degenerative disease. Spine (Phila Pa 1976) 33:903-908, 2008

27. Zaidi Q, Danisa OA, Cheng W: Measurement techniques and utility of Hounsfield unit values for assessment of bone quality prior to spinal instrumentation: a review of current literature. Spine (Phila Pa 1976) 44:E239-E244, 2019

28. Zou D, Li W, Deng C, Du G, Xu N: The use of CT Hounsfield unit values to identify the undiagnosed spinal osteoporosis in patients with lumbar degenerative diseases. Eur Spine J 28:1758-1766, 2019

29. Zou D, Li W, Xu F, Du G: Use of Hounsfield units of S1 body to diagnose osteoporosis in patients with lumbar degenerative diseases. Neurosurg Focus 46(5):E6, 2019

\section{Disclosures}

The authors report no conflict of interest concerning the materials or methods used in this study or the findings specified in this paper.

\section{Author Contributions}

Conception and design: Li, Zou. Acquisition of data: Zou, Muheremu. Analysis and interpretation of data: Zou, Muheremu. Drafting the article: Li, Zou, Muheremu. Critically revising the article: Zou. Reviewed submitted version of manuscript: all authors. Approved the final version of the manuscript on behalf of all authors: Li. Statistical analysis: Zou, Muheremu. Administrative/technical/material support: Li, Sun, Zhong, Jiang. Study supervision: Li.

\section{Correspondence}

Weishi Li: Peking University Third Hospital, Beijing, China. puh3liweishi@163.com. 